hospital causes. Overall 8 cases out of 29 (27.6\%) scored between 2.4 and 2.8 (1=Definitely avoidable, $2=$ Strong evidence of avoidability), 8 cases (27.6\%) scored between 3.0 and 4.6 ( $3=$ Probably avoidable, $4=$ Possibly avoidable), and the remaining 13 cases $(44.8 \%)$ between 4.0 and $5.8 \quad(5=$ Slightly avoidable or $6=$ Definitely not avoidable). Variation between raters was satisfactory with ICC 0.84 (95\% CI: 0.73 to 0.92). Common themes among cases with strong evidence of avoidability were symptoms or physical findings indicating a potentially serious condition and refusal by patients or their carers to be transported to hospital. RCRRs require linked ambulance, hospital and mortality data to ensure accurate assessment in light of the diagnosis and cause of death.

Conclusion Retrospective case record reviews (RCRR) have been widely used to assess quality of care but evidence for their use in prehospital ambulance settings is limited. We aimed to review case records of potentially avoidable deaths related to ambulance care.

Conflict of interest None

Funding National Institute for Health Research, UK.

\section{TEMPORAL TRENDS IN SURVIVAL OF PATIENTS WITH AND WITHOUT DIABETES FOLLOWING OUT-OF- HOSPITAL CARDIAC ARREST: A NATIONWIDE DANISH STUDY}

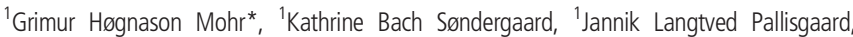
${ }^{1}$ Sidsel Gamborg Møller, ${ }^{1,2}$ Mads Wissenberg, 1,2 Lena Karlsson, ${ }^{3}$ Steen Møller Hansen, ${ }^{3,4}$ Kristian Kragholm, ${ }^{5}$ Lars Køber, ${ }^{2}$ Freddy Lippert, ${ }^{1,2}$ Fredrik Folke, ${ }^{7,8}$ Tina Vilsbøll, ${ }^{3,4,6}$ Christian Torp-Pedersen, ${ }^{1,8,9,10}$ Gunnar Gislason, ${ }^{1}$ Shahzleen Rajan. ${ }^{1}$ Department of Cardiology, Copenhagen University Hospital Gentofte, Copenhagen, Denmark; ${ }^{2}$ Emergency Medical Services Copenhagen, University of Copenhagen, Denmark; ${ }^{3}$ Unit of Epidemiology and Biostatistics, Aalborg University Hospital, Aalborg, Denmark; ${ }^{4}$ Department of Cardiology, Aalborg University Hospital, Aalborg, Denmark; ${ }^{5}$ The Heart Centre, Copenhagen University Hospital, Rigshospitalet, Copenhagen, Denmark; ${ }^{6}$ Department of Health Science and Technology, Aalborg University, Denmark; ${ }^{7}$ Clinical Metabolic Physiology, Steno Diabetes Centre Copenhagen, University of Copenhagen, Copenhagen, Denmark; ${ }^{8}$ Department of Clinical Medicine, Faculty of Health and Medical Sciences, University of Copenhagen, Copenhagen, Denmark; ${ }^{9}$ National Institute of Public Health, University of Southern Denmark, Copenhagen, Denmark; ${ }^{10}$ The Danish Heart Foundation, Copenhagen, Denmark

\subsection{6/10.1136/bmjopen-2018-EMS.21}

Aim In Denmark, survival from out-of-hospital cardiac arrest (OHCA) has increased markedly following national initiatives to improve cardiopulmonary resuscitation (CPR). ${ }^{1,2,3}$ However, whether the temporal improvement in OHCA survival also applied for diabetics is unknown.

Method Patients $>18$ years with OHCA of presumed cardiac cause were identified from the Danish Cardiac Arrest Registry during 2001-2014. Patients with prescriptions for glucose-lowering drugs up to 180 days before OHCA were identified as diabetics. Associations between diabetes and return of spontaneous circulation (ROSC) upon hospital arrival and 30 day survival were estimated with logistic regression adjusted for patient- and OHCA-related characteristics. In total, 28,955 OHCA cases were included and $4276(14.8 \%)$ of those were diabetics. Compared to non-diabetics, diabetics had higher prevalence of comorbidity, same prevalence of bystander witnessed arrests $(42.0 \%$ vs $43.2 \%)$ and bystander CPR $(51.7 \%$ vs $52.7 \%)$, more arrests in residential locations $(77.3 \%$ vs $73.0 \%)$ and were less likely to have initial shockable heart rhythm (23.5\% vs 27.9\%). From 2001 to 2014, temporal increases of ROSC (from $8.8 \%$ to $22.3 \%$ among diabetics vs
$7.8 \%$ to $25.7 \%$ among non-diabetics) and 30 day survival (from $2.8 \%$ to $9.7 \%$ among diabetics vs $3.5 \%$ to $14.8 \%$ among non-diabetics) were observed in both patient groups (P for trends $<0.001)$. Diabetes was associated with decreased probability of achieving ROSC (odds ratio (OR) 0.75 [95\% confidence interval (CI) 0.67 to 0.84$]$ ) as well as 30 day survival (OR 0.57 [95\% CI: 0.47 to 0.68 ]).

Results ROSC and 30 day survival increased in patients with and without diabetes. However, diabetes was associated with lower odds of ROSC and 30 day survival.

\section{REFERENCES}

1. Hansen SM, Hansen CM, Folke F, et al. Bystander defibrillation for out-of-hospital cardiac arrest in public vs residential locations. JAMA Cardiol 2017:2(5):507-514. doi:10.1001/jamacardio.2017.0008

2. Wissenberg $M$, Lippert FK, Folke $F$, et al. Association of national initiatives to improve cardiac arrest management with rates of bystander intervention and patient survival after out-of-hospital cardiac arrest. Jama 2013;310:1377-1384. doi:10.1001/jama.2013.278483

3. Hansen SM, Wissenberg M, Rajan S, et al. Rapport fra dansk hjertestopregister 2001-2014. http://genoplivning.dk/wp-content/uploads/2016/05/Rapport-fraDansk-Hjertestopregister-2001-2014.pdf [Accessed: 7 June 2017].

Conflict of interest None

Funding None

\section{PRESENCE OF FAMILY IN RESUSCITATION IN PREHOSPITAL: THE PHYSICIANS' AND NURSES' OPINION}

${ }^{1} \mathrm{~T}$ Amaral ${ }^{*},{ }^{2} \mathrm{M}$ Santos, ${ }^{3} \mathrm{~N}$ Gaibino, ${ }^{2} \mathrm{~N}$ Santos, ${ }^{4} \mathrm{P}$ Caldeira, ${ }^{5} \mathrm{M}$ Job. ${ }^{1}$ Hospital São José (CHLC, EPE), Lisboa, Portugal; ${ }^{2}$ Hospital Fernando Fonseca, Amadora, Portugal; ${ }^{3}$ Hospital Santa Maria (CHLN, EPE), Lisboa, Portugal; ${ }^{4}$ Hospital Cascais, Cascais, Portugal; ${ }^{5}$ Hospital Garcia Orta, Almada, Portugal

\subsection{6/10.1136/bmjopen-2018-EMS.22}

Aim The family's presence in resuscitation is a current topic but we have a lack of studies in Portugal. In prehospital setting, the patient's home is the most often place where is supplied resuscitation care.

Method We applied an online questionnaire to 116 nurses and physicians who work in prehospital setting.

Results Of the 116 professionals surveyed, 49 were physician's $(42,7 \%)$ and $67(57,3 \%)$ were nurses. At mobile medical car work 93 professionals: 44 nurses $(65,7 \%)$ and 49 physicians $(100 \%)$. From the remaining nurses, $18(26,9 \%)$ work at an immediate life support ambulance and the other $5(7,5 \%)$ at another resource. All the professionals said they provided care in the prehospital with the presence of family members. 31\% have a favourable opinion (12,3\% physicians vs $44,8 \%$ nurses) about the presence of family members and $49,3 \%$ have an unfavourable opinion ( $71,4 \%$ physicians vs $32,9 \%$ nurses). They said that the main advantages are obtaining the health history, recognition of the team effort and facilitating the grieving process. The disadvantages there may be interference from family in procedures, the stress experienced by the team for being watched and anxiety felt by the family.

Conclusion Most nurses have a positive opinion about the presence of family members during resuscitation as long as they are able to perceive the significance of interventions in resuscitation situations and do not interfere with team performance. On the other hand, most physicians have an opinion against, mainly due to the distraction of the team during all procedures and the trauma to the family with the invasive procedures.

Conflict of interest None

Funding None 